\title{
A modulatory effect of male voice pitch on long-term memory in women: evidence of adaptation for mate choice?
}

\author{
David S. Smith • Benedict C. Jones • \\ David R. Feinberg $\cdot$ Kevin Allan
}

Published online: 8 September 2011

(C) Psychonomic Society, Inc. 2011

\begin{abstract}
From a functionalist perspective, human memory should be attuned to information of adaptive value for one's survival and reproductive fitness. While evidence of sensitivity to survival-related information is growing, specific links between memory and information that could impact upon reproductive fitness have remained elusive. Here, in two experiments, we showed that memory in women is sensitive to male voice pitch, a sexually dimorphic cue important for mate choice because it not only serves as an indicator of genetic quality, but may also signal behavioural traits undesirable in a long-term partner. In Experiment 1, we report that women's visual object memory is significantly enhanced when an object's name is spoken during encoding in a masculinised (i.e., lower-pitch) versus feminised (i.e., higher-pitch) male voice, but that no analogous effect occurs when women listen to other women's voices. Experiment 2 replicated this pattern of results, additionally showing that lowering and raising male voice pitch enhanced and impaired women's memory, respectively, relative to a baseline (i.e., unmanipulated) voice condition. The modulatory effect of sexual dimorphism cues in the male voice may reveal a mate-choice adaptation within women's memory, sculpted by evolution in response to the dilemma posed by the double-edged qualities of male masculinity.
\end{abstract}

D. S. Smith • B. C. Jones $\cdot$ K. Allan $(\square)$

School of Psychology, College of Life Sciences and Medicine,

University of Aberdeen,

Aberdeen AB24 2UB, UK

e-mail:k.allan@abdn.ac.uk

D. R. Feinberg

Department of Psychology, Neuroscience and Behaviour,

McMaster University,

Hamilton, Ontario L8S 4K1, Canada
Keywords Memory · Social cognition · Speech perception

Tulving (2002) pointed out that the evolutionary origins of human memory are quite obscure. Nairne and colleagues have suggested (e.g., Nairne \& Pandeirada, 2008) that this is largely because research tends to focus on how memory operates, while ignoring the functional, or adaptive, benefits contingent upon its operation. Addressing the same issue in an earlier functionalist review of memory systems research, Sherry and Schacter (1987) suggested that human memory might contain mechanisms that originated as solutions to particular ancestral environmental problems that have since exapted as solutions to relatively more recent problems. Consequently, evidence of the original evolved design may still be obtained under the right circumstances. But what are these circumstances? Nairne and Pandeirada argued that memory mechanisms shaped by evolution to solve particular problems may reveal themselves via their sensitivity to adaptively important information. In keeping with evolutionary theory (e.g., Endler, 1986), Nairne and Pandeirada emphasised particular domains, including survival, navigation, social exchange, kin recognition, and reproduction, where specialised mechanisms sensitive to adaptively important content might exist within memory. To support this functionalist approach, Nairne and Pandeirada reviewed evidence for enhanced recall of information encoded in relation to survival in grasslands versus urban contexts, a robust effect that has attracted considerable interest (e.g., Kang, McDermott, \& Cohn, 2008; Klein, Robertson, \& Delton, 2010; Weinstein, Bugg, \& Roediger, 2008; but see Soderstrom \& McCabe, 2011). Here, to extend the empirical basis for a functionalist approach, we focus on a specific problem faced by women engaging in mate-choice decisions, and we provide evi- 
dence suggesting that this problem may have led to specialisation within long-term memory function.

Prior studies have examined memory function within the context of relationships, looking at the effects of factors such as jealousy and infidelity (Schutzwohl \& Koch, 2004). To our knowledge, however, specific links between women's memory and mate-choice-relevant cues in men have never been demonstrated. In fact, evidence of such links has proven to be surprisingly elusive (e.g., Anderson et al., 2010; Becker, Kenrick, Guerin, \& Maner, 2005; Maner et al., 2003; but see van Wingen, Mattern, Verkes, Buitelaar, \& Fernandez, 2008, for evidence of changes in hippocampal activation in women viewing male vs. female faces). Our approach here is to focus on a well-known and reasonably well-understood problem within women's mate choice. This problem concerns how women evaluate cues of sexual dimorphism (i.e., masculinity) in men, as an indicator of mate quality. Masculine male traits signal the desirable attribute of heritable immunity to infection, are associated with other putative cues of long-term health (e.g., high facial and body symmetry; Gangestad \& Thornhill, 2003; Little et al., 2008), and also with reduced incidence of illness (Rhodes, Chan, Zebrowitz, \& Simmons, 2003; Thornhill \& Gangestad, 2006). Male masculinity is also positively correlated with both reported reproductive success in a natural fertility population (Apicella, Feinberg, \& Marlowe, 2007) and indices of mating success in samples of undergraduate students (Hughes, Dispenza, \& Gallup, 2004; Puts, 2005; Rhodes, Simmons \& Peters, 2005).

However, biological indicators of genetic quality coexist in relatively masculine individuals with less desirable behavioural traits, including a higher likelihood of marital problems and divorce (Booth \& Dabbs, 1993), perceived antisocial traits (e.g., dishonesty, lack of emotional warmth; Perrett et al., 1998), and an increased interest in shortversus long-term relationships (Boothroyd et al., 2008; Rhodes et al., 2005). Thus, any fitness-related benefits from mating with a relatively masculine male may trade off against the costs of their behavioural tendencies within a long-term relationship (Fink \& Penton-Voak, 2002; Gangestad \& Simpson, 2000). Consistent with this idea, women's preferences for male masculinity are modulated by a wide variety of factors, including menstrual cycle phase and hormone levels (e.g., Feinberg et al., 2006; Puts, 2005), hormonal contraceptive use (Feinberg, DeBruine, Jones, \& Little, 2008), whether or not one is breastfeeding (Apicella \& Feinberg, 2009), and indices of a women's own attractiveness (Vukovic et al., 2010; Vukovic et al., 2008).

Masculine characteristics therefore appear to be equivocal with regard to the quality of a specific man as a potential partner. Because of this, it has been argued
(Bateson \& Healy, 2005) that reliance on masculinity cues as an absolute indicator of mate quality could lead women towards costly - that is, maladaptive - mate choices. Bateson and Healy concluded that cognitively elaborate comparative mate-choice mechanisms, allowing currently available potential mates to be evaluated against alternative mates, are needed for adaptive mate choice (see Sedikides, Ariely, \& Olsen, 1999, for evidence of comparative evaluation in young healthy adults). The essence of this proposal is that to help mediate the trade-off involved in choosing a masculine partner, memory is needed to rapidly provide information about the attributes and past behaviour of that individual and their competitors for our affections.

\section{The present study}

Our experiments therefore investigate whether women's memory for details associated with men is modulated by the degree to which the men display sexually dimorphic traits indicative of high genetic quality. Translated into the functionalist framework provided by Nairne and colleagues, our experiment asks whether women's memory is sensitive to the presence of masculinity cues in men that signal adaptively valuable information for mate choice. The specific prediction that we test is whether a manipulation of men's vocal cues indicating enhanced versus reduced sexual dimorphism can modulate women's memory for details of concurrently presented object images. We chose to employ a manipulation of sexually dimorphic vocal characteristics in the present study because women's preferences for vocal cues of sexual dimorphism in the opposite sex (i.e., lower pitch in men) are stronger and less individually variable than preference for cues of facial sexual dimorphism (e.g., Collins, 2000; Feinberg et al., 2006; Feinberg, Jones, Little, Burt, \& Perrett, 2005). Indeed, the presence of strong individual differences in women's preferences for men's faces might explain why prior work has been unable to demonstrate associated effects upon the accuracy of women's memory (e.g., Anderson et al., 2010; van Wingen et al., 2008).

Using computer-based pitch manipulation techniques (Feinberg et al., 2005), we produced masculinised (i.e., lower-pitch) and feminised (i.e., higher-pitch) versions of auditory recordings taken from young healthy adult men and women (see the Method sections for details). Each speaker was asked to say the names of 64 different animate and inanimate objects. From this set of 64 objects, we randomly picked a subset of 32 to show to each participant while she concurrently listened to the name of each object played over headphones. Half of the names were spoken in a male voice and half in a female voice, and within each voice sex, half of the sound clips were masculinised and 
half were feminised. Memory for studied objects was then assessed as a function of voice sex and pitch (raised vs. lowered) at study, using a two-alternative forced choice recognition task (2AFC) that required images of studied objects to be discriminated from similar unstudied foil images (see Fig. 1). Importantly, to verify that the pitch manipulation affected the participants' perceptions of attractiveness, following the recognition task we measured overt preferences for sexually dimorphic male and female vocal characteristics using sound clips of studied and also unstudied object names. At issue was whether voice pitch cues that indicate sexual dimorphism could affect memory for details that co-occur with opposite-sex men's voices but not with within-sex women's voices.

\section{Experiment 1}

\section{Method}

Participants We employed 45 female participants split into two groups according to their reported hormonal contraceptive use, giving 25 nonusers (mean age $=21.3$ years, $S D=$ 4.1 , range $18-38$ years) and 20 users (mean age $=20.1$ years, $S D=1.7$, range 18-23 years). We examined hormonal contraceptive use as a between-subjects factor because of prior evidence for different masculinity preferences in women using or not using hormonal contraceptives (see Feinberg et al., 2008). It should be noted, however, that strong preferences for masculine characteristics in men's voices are generally still observed even in women using hormonal contraceptives (Feinberg et al., 2008; Vukovic et al., 2008). All participants reported sexual preferences for members of the opposite sex.

Stimuli From four young adult men and four young adult women we recorded auditory clips of 64 different object names taken from a set of images within a commercially available photo clip-art image database (Hemera Technologies, Inc.). Two closely matching but not identical examples of each type of object were chosen from the image database, and each individual object image was then superimposed on a white background, giving us 128 different image files in total (for examples, see Fig. 1). Individual auditory clips were digitally manipulated to enhance or reduce each speaker's sexually dimorphic vocal traits. All acoustic measurements and manipulations were made using the Praat software, version 5103 (Boersma \& Weenink, 2010, www.praat.org). For each clip, the speaker's voice pitch was either raised or lowered using the pitch-synchronous overlap add algorithm (PSOLA), a method employed in numerous studies on vocal attractiveness (Feinberg et al., 2006; Feinberg et al., 2005; Vukovic et al., 2008). The PSOLA method alters a speaker's pitch while all other aspects of the recording remain unaffected by the manipulation (e.g., the speech rate and formant frequency), meaning that preferences for one manipulated clip versus another from the same speaker can be attributed to alterations in vocal pitch. To exaggerate the sexual dimorphism of the speakers' voices, the frequency of each clip was raised or lowered by \pm 0.5 equivalent rectangular bandwidths (ERBs) of the original frequency. This manipulation is approximately equivalent to $20 \mathrm{~Hz}$ at a centre frequency of approximately $120 \mathrm{~Hz}$ (the average male voice
Fig. 1 Examples of learning (left) and recognition trials (right). During learning trials, each participant viewed a single object image while listening to the name of the object spoken by a male or female voice either raised or lowered in pitch. During recognition trials, two versions of each previously viewed object type were shown, and participants had to indicate which image had been studied

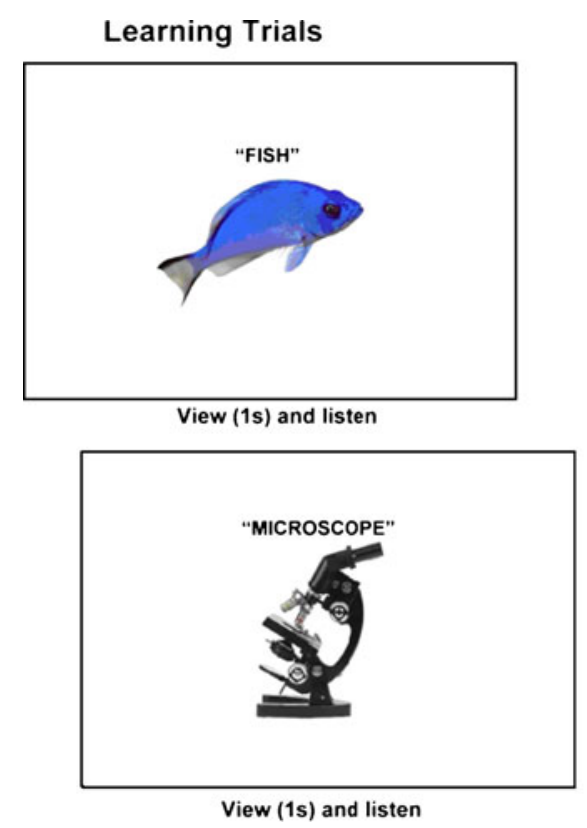

View (1s) and listen

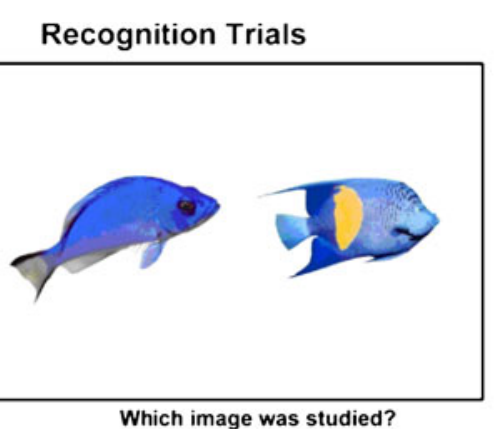

Which image was studied?

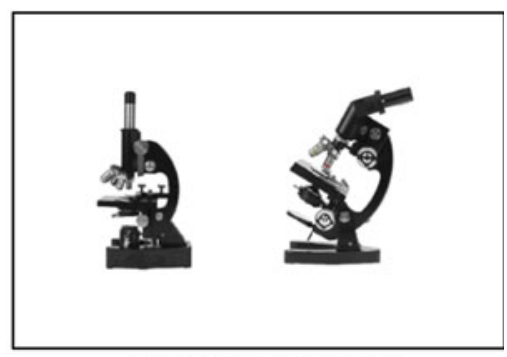

Which image was studied? 
pitch; Childers \& Wu, 1991), taking into account the logarithmic scale of pitch perception (Traunmüller, 1990). The \pm 0.5 ERB manipulation has previously been shown to alter women's ratings of male vocal attractiveness using various kinds of auditory content (e.g., Feinberg et al., 2006; Feinberg et al., 2005; Vukovic et al., 2008). The average unmanipulated pitch of the recordings taken from our four male speakers was $133 \mathrm{~Hz}(S D=19.1)$, and the average pitch of the four female speakers was $207 \mathrm{~Hz}$ $(S D=18.7)$.

The study phase consisted of 32 trials. During each trial, one version of an object image was shown while its name was simultaneously played over headphones. The sex and voice pitch (i.e., raised vs. lowered) of each voice clip was controlled such that each participant heard 16 male and 16 female voices, and within each sex 8 voices had lowered and 8 voices had raised pitch. Within each of the eight clips belonging to the four voice conditions (i.e., male lowered, male raised, female lowered, female raised), we employed two clips from each of our four male and four female speakers. Across participants, we differently randomised the allocation of specific speakers to each of the four voice conditions. We also randomly picked a set of 32 objects (from the set of 64) to be studied, and we randomly allocated these to the four different voice conditions. In addition, we counterbalanced which particular object image (from the pair of images) was studied, such that half of the participants studied one version, while the remaining half studied the other version.

During each of the 32 recognition trials, object memory was tested by presenting both examples of each kind of studied object, and participants were asked to indicate using their computer mouse which example was studied (2AFC recognition). The side of onscreen presentation was randomised from trial to trial for studied and unstudied images. Trial order was also randomised differently for each participant during the study and recognition phases.

On each voice preference test trial, we played both auditory clips (i.e., the raised- and the lowered-pitch versions) for one of 64 objects (32 studied and 32 unstudied). These comprised 32 male voice and 32 female voice (16 enhanced and 16 reduced in sexual dimorphism), using clips from each of the four male and four female speakers equally often.

Procedure Each participant initially completed a consent form that contained a cover story suggesting that the experimental goal was to examine sociosexual orientation and object preference. Our intention was to mask the fact that the experiment involved a test of memory, and also to ensure that the participants would pay close attention to each object. No overt response was required during each study trial, which began with a 2 -s blank screen, followed by presentation of each image for $1 \mathrm{~s}$, accompanied simultaneously by a recording of the object's name played over headphones. The study phase lasted approximately $2 \mathrm{~min}$, and then each participant was asked to complete a Sudoku puzzle for $5 \mathrm{~min}$. Following this study-test interval, the participants were informed that we would now examine their ability to remember objects that they had viewed. Participants were told that they would see two images shown side by side on the screen, and their task was simply to click on the image of the studied object using the computer mouse. This phase of the experiment was selfpaced and lasted on average for $2 \mathrm{~min}$.

Following the memory test, we asked the participants to perform an auditory vocal preference test. On each preference trial, two onscreen icons were shown, one on each side of the screen, and participants were free to click first on either one of the icons. When they did so, the first auditory clip was played, and then the participants were instructed to click on the other icon to play the other auditory clip. Each click resulted in one clip of a given object name being played that was either the lowered- or raised-pitch version of an object name, both spoken by the same speaker, either male or female. Both clips in each trial had to be played before a preference judgement could be made, and the allocation of the lowered- and raised-voice pitch clips to the left or right screen icons was randomised for each trial. In total, each participant provided us with 64 preference judgements using a 7-point Likert-like scale (Vukovic et al., 2008) upon 32 studied and 32 unstudied object names. Across the 64 preference trials, we blocked the sex of speakers' voices and also whether the clips were of studied versus unstudied objects. Thus, we in effect gave four different preference tests, and each participant had a different random ordering of these blocked factors. Upon completing the experiment, the participants were thanked for their participation and debriefed.

\section{Results and discussion}

Voice preference To ensure that the pitch manipulations on the stimuli newly created for our experiment produced a robust effect on vocal attractiveness, we first of all examined the preference data to determine whether participants showed the expected overall pattern of preference for heightened vocal sexual dimorphism in members of the opposite sex (i.e., a preference for vocal masculinity in men). Responses on each trial were coded on a 7-point scale, where a mean score within each condition of higher than 3.5 indicates a preference for exaggerated sexually dimorphic vocal traits. The mean preference ratings are shown in Fig. 2, separately for studied and unstudied object names. Analysis of these data showed that preferences for masculi- 


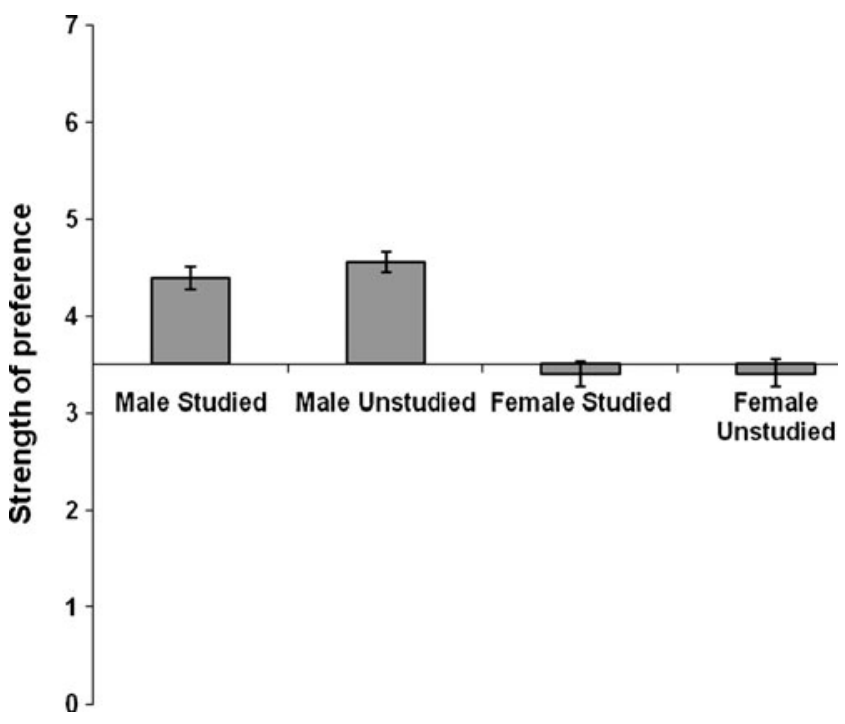

Fig. 2 Mean $(N=45)$ strength of preference for sexually dimorphic voice pitch in Experiment 1-that is, lowered pitch in male speakers and raised pitch in female speakers $( \pm 1 S E$ of the mean). The data are shown separately for studied and unstudied object names. A score of 3.5 (where the $x$-axis is placed) indicates chance (i.e., no preference), and a score higher than 3.5 indicates preference for sexually dimorphic voice pitch

nised male voices were significantly higher than chance (i.e., $3.5)$ [studied, $M=4.39(S D=0.79), t(44)=7.61, p<.001$; unstudied, $M=4.56(S D=0.74), t(44)=9.68, p<.001]$. No significant preferences for heightened femininity in female voices were observed, for either the studied or unstudied object names [maximum $t(44)=-0.632, p=.531$ ]. A mixeddesign ANOVA on female participants' preference data examined whether hormonal contraceptive use was associated with different preferences. The ANOVA indicated no effects of the between-subjects contraceptive use factor on preferences for masculinity in male voices or femininity in female voices [maximum $F(1,44)=2.58, p=.115$ ].

Object memory The pattern of object memory as a function of voice sex and pitch (raised vs. lowered) is shown in Fig. 3. As predicted, participants' object memory was sensitive to the pitch of male voices-memory was better for objects initially spoken in lowered (i.e., masculinised) vs. raised (i.e., feminised) pitch male voices. Mixed-design ANOVA [between-subjects factor: hormonal contraceptive use; within-subjects factors: voice sex (male/female) and voice pitch (raised/lowered)] was used to examine whether the voice pitch manipulation affected memory per se, or whether the effect of the pitch manipulation interacted with speaker's sex. Note that this way of coding the factors allowed us to check whether lowered voice pitch affected memory regardless of the speaker's sex. The ANOVA indicated no effects of the between-subjects contraceptive use factor $[F(1,43)=$ $0.657, p=.422]$, speaker's sex $[F(1,43)=0.81, p=.38]$, or

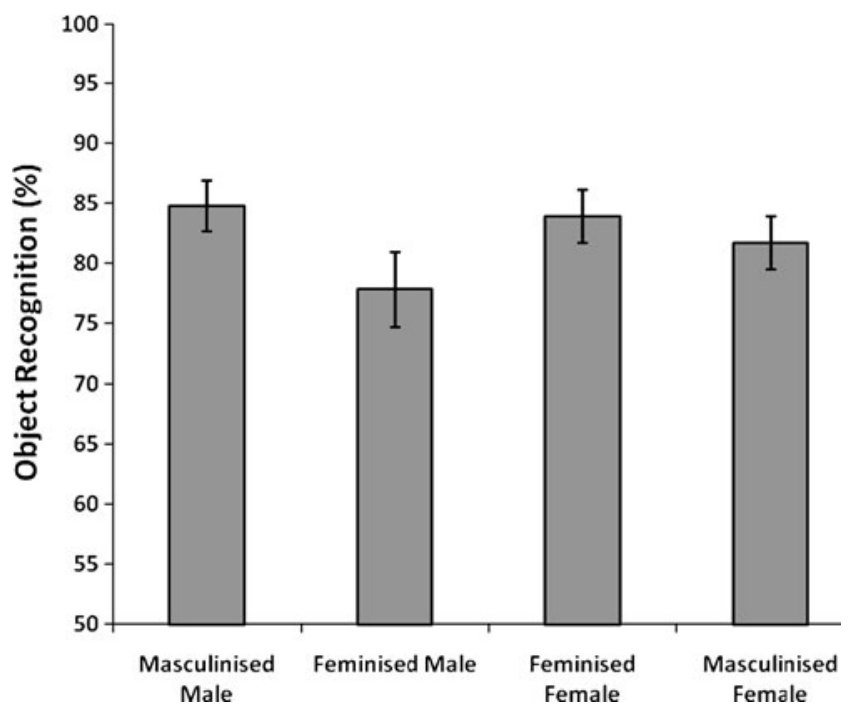

Fig. 3 Mean percentages of correct object recognition $( \pm 1 S E$ of the mean) according to the different voice conditions at study in Experiment 1. Masculinised and feminised male voices were lowered versus raised in pitch, respectively, while feminised and masculinised female voices were raised versus lowered in pitch, respectively

raising/lowering vocal pitch $[F(1,43)=1.27, p=.27]$. But a significant interaction between voice sex and pitch was obtained $\left[F(1,43)=6.86, p=.012, \eta_{\mathrm{p}}^{2}=.138\right]$. Pairedsamples $t$ tests confirmed that object memory was significantly better when object names were spoken by male voices lowered in pitch versus those raised in pitch $[84.7 \%$ $(S D=14.1)$ vs. $77.8 \%(S D=21.1) ; t(44)=2.36, p=.022]$, but no such effect was found for objects named by female voices $[83.9 \%(S D=14.9)$ vs. $81.7 \%(S D=14.7)$; $t(44)=0.94, p=.35]$.

The analyses above revealed that participants' memories were sensitive to whether object names were spoken initially by lowered (i.e., masculinised) versus raised (i.e., feminised) male voices. In contrast, the sexual dimorphism of female voice clips had no detectable effect on object memory. We are therefore confident that the modulatory effect of male voice pitch on women's memory did not reflect an effect of lowering voice pitch per se. The pattern of the memory effect also mirrored the pattern of voice preference, insofar as our female participants had selective preferences for male vocal masculinity and not for female vocal femininity (see also Feinberg et al., 2006). These findings precisely match our prediction that memory in women may be sensitive to the presence of men's voice cues indicative of enhanced versus reduced sexual dimorphism.

We therefore conclude, because of the importance of male sexual dimorphism for women's mate choice (e.g., Apicella et al., 2007; Dabbs \& Mallinger, 1999; Feinberg et al., 2005), that our findings are consistent with the existence of a specialised encoding mechanism sensitive 
to male voice pitch. But, should we interpret the modulatory effect of male voice pitch as a specific enhancement of women's memory for information that co-occurs with signals of preferred - that is, masculinised-attributes? Alternatively, have we revealed that women's memory is specifically impaired for information that co-occurs with signals of less preferable - that is, feminised - attributes in men? Or, does the effect of male voice pitch on women's memory reflect a combination of enhancements and impairments?

To address this issue, in Experiment 2 we once again used the 2AFC procedure to examine the modulatory effect on women's memory of lowering and raising men's voice pitch, but this time we included unmanipulated as well as manipulated pitch recordings. If the modulatory effect of lowering and raising male voice pitch on women's memory arises from a corresponding pattern of enhancement and impairment, performance in the baseline condition should then sit squarely in the middle - that is, memory in the lowered-pitch condition should be better than baseline, while memory in the raised-pitch condition should be worse than baseline. The aim of Experiment 2 was to determine whether such a linear pattern was present. In addition, we included manipulated and unmanipulated women's voices in order to confirm, by replication, that memory modulations remain specific to opposite-sex (men's) voices.

\section{Experiment 2}

\section{Method}

Participants We recruited 46 female participants, all of whom reported sexual preferences for members of the opposite sex. According to their self-reported hormonal contraceptive use, the group comprised 28 pill users (mean age $=20.1$ years $(S D=1.5)$, range $18-25$ years) and 18 nonusers (mean age $=19.7$ years $(S D=1.7)$, range $18-24$ years).

Stimuli The stimuli were identical to those used in Experiment 1, except for the inclusion of the unmanipulated vocal recordings from each of the four male and four female speakers.

Procedure The experiment was procedurally identical to Experiment 1, with the following exceptions. During encoding, participants were presented with 48 rather than 32 trials. The additional 16 trials comprised eight unmanipulated male voices (two from each of the four male speakers) and eight unmanipulated female voices (two from each of the four female speakers), paired up with a corresponding object image. The retrieval phase also employed 48 rather than 32 trials, on which pairs of object images were presented for $2 \mathrm{AFC}$ recognition of the studied object. For the voice preference test that followed, participants either performed a preference judgement involving masculinised versus feminised voices, or they judged whether they preferred an unmanipulated voice versus a manipulated voice. In total, they gave 64 preference judgements (32 male, 32 female). Within each voice gender, 16 judgements involved masculinised versus feminised recordings of the same object name, and 16 involved unmanipulated versus manipulated recordings of the same object name (eight masculinised and eight feminised). As was the case in Experiment 1, sex of voice was a blocked factor. In addition, we blocked whether each trial solely contained manipulated recordings or a combination of manipulated and unmanipulated recordings. This resulted in four blocks within the preference phase, which were given in a different randomised order to each participant. All other counterbalancing and randomising factors were as per the preference tests in Experiment 1.

\section{Results and discussion}

Voice preference First of all, we examined the preference data to determine whether participants once again showed preferences for vocal sexual dimorphism specifically in men and not women. The mean preference ratings are shown in Fig. 4, separately for the trials involving masculinised versus feminised voice recordings and for the trials comprising unmanipulated versus manipulated recordings. For convenience, we will refer to these trial types as "standard" versus "nonstandard," respectively, to reflect the fact that unmanipulated stimuli have not

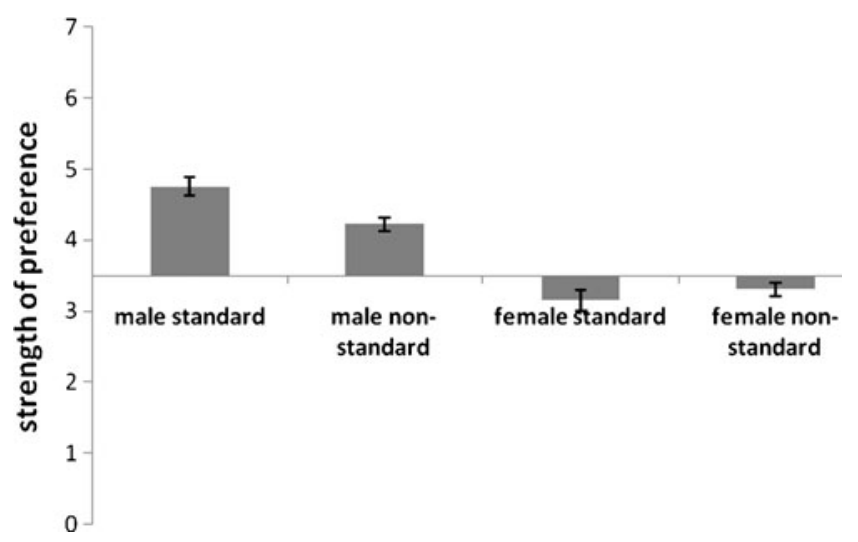

Fig. 4 Mean $(N=46)$ strength of preference for sexually dimorphic voice pitch in Experiment 2-that is, lowered pitch in male speakers and raised pitch in female speakers ( $\pm 1 S E$ of the mean). The data are shown separately for the "standard" (masculinised vs. feminised pitch) and the "nonstandard" (unmanipulated vs. manipulated pitch) tests 
typically been used within the literature employing computer transformation techniques to examine preferences for sexual dimorphism, either in vocal or in facial stimuli.

Figure 4 clearly shows a preference for masculinised male voices that is present in both the standard and nonstandard preference trials, and furthermore that preference strength is reduced in the nonstandard trials. Analysis of these data revealed that preferences for masculinised male voices were significantly higher than chance (i.e., 3.5) [standard, $4.76(S D=0.83), t(45)=10.25, p<.001$; nonstandard, $4.22(S D=0.62), t(44)=7.86, p<.001]$. Paired-samples $t$ tests confirmed that male voice sexual dimorphism preference was significantly higher in the standard versus the nonstandard trials $[t(45)=5.78, p<$ $.001]$. In contrast to the male voice data, there were no significant preferences for heightened femininity in women's voices, in either the standard or nonstandard trials [maximum preference rating was $3.15(S D=1.00), t(45)=2.33 p=.24]$, and neither was there an effect of standard versus nonstandard trial type. We also carried out a mixed-design ANOVA on the preference data to examine whether these were altered by hormonal contraceptive use. The ANOVA indicated no effects of the between-subjects contraceptive use factor on preferences for masculinity in male voices, or upon preferences for femininity in female voices [maximum $F(1$, $44)=2.58, p=.11]$. In sum, the pattern of preferences replicates that obtained in Experiment 1.

Object memory Figure 5 shows correct 2AFC object memory as a function of voice sex and pitch at encoding. Women's object memory appears once again to be selectively sensitive to men's voice pitch and to be unaffected by the manipulation of pitch in women's voices. Specifically, memory appears to be better for objects initially spoken in

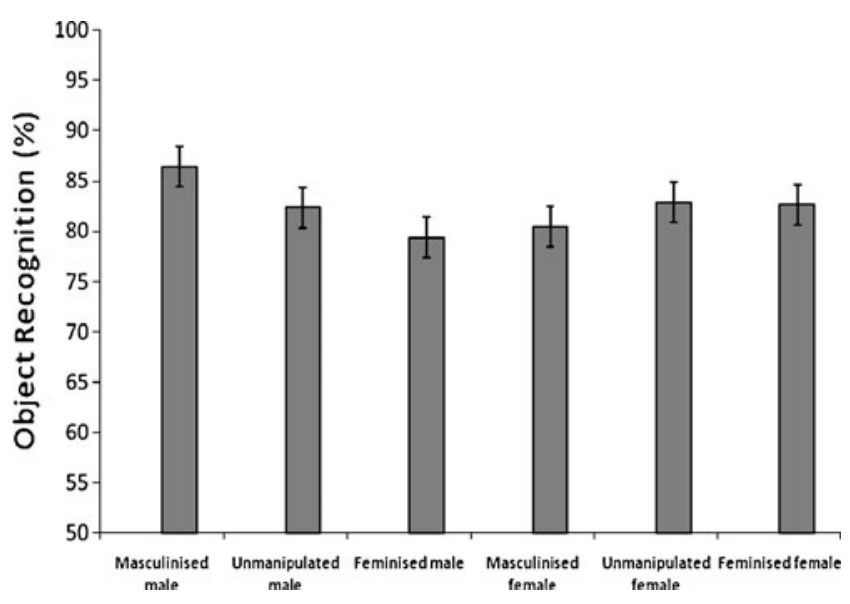

Fig. 5 Mean percentages of correct object recognition $( \pm 1 S E$ of the mean) according to voice sex and pitch (raised, lowered, or unmanipulated) at encoding in Experiment 2. See the Method section for details of the pitch manipulations lowered versus unmanipulated men's voices, but worse for the raised-pitch men's voices versus unmanipulated voices. First of all, we verified that including the unmanipulated voices did not alter the basic pattern obtained in Experiment 1. We therefore analysed data from the manipulated pitch conditions using the same mixed-design ANOVA employed in Experiment 1 [between-subjects factor: hormonal contraceptive use; within-subjects factors: voice sex (male/female) and voice pitch (raised/lowered)]. A voice sex $\mathrm{x}$ pitch interaction was present $\left[F(1,44)=5.87, p=.02, \eta_{\mathrm{p}}^{2}=\right.$ $.118]$, which was driven by an effect of pitch on the men's voices [lowered, $86.4 \%(S D=14.9)$ vs. raised, $79.3 \%(S D=$ $16.9) ; t(45)=3.14, p=.003$ ] but not the women's voices [lowered, $80.4 \%(S D=17.2)$ vs. raised, $82.6 \%(S D=15.7)$; $t(45)=0.75, p=.46]$. This pattern exactly replicated the one obtained in Experiment 1.

Having established that we could replicate the oppositesex specific memory modulation due to voice pitch, we next examined effects of voice sex and pitch relative to the baseline (i.e., unmanipulated) conditions. Within the same ANOVA structure described above, but with an additional level in the pitch factor for the unmanipulated recordings, the voice sex $\mathrm{x}$ pitch interaction approached significance $[F$ $(2,88)=2.75, p=.07]$, while the associated contrast testing for linear effects within the interaction was significant $[F(1$, $44)=5.87, p=.02]$. This outcome suggests that a linear effect of pitch was specific to men's voices. To confirm this, we ran two final one-way ANOVAs on the 2AFC performance data to examine pitch effects (raised, lowered, unmanipulated) within each sex of voice. A significant pitch effect and a significant linear contrast effect were present for men's voices $\left[F(2,90)=4.00, p=.022, \eta_{\mathrm{p}}^{2}=\right.$ .082 , and $F(1,45)=9.83, p=.003, \eta_{\mathrm{p}}{ }^{2}=.179$, respectively], but neither were present for the women's voices (both $F<1$ ).

The aim of Experiment 2 was to determine whether the modulatory effect of men's voice pitch was due to the memory-enhancing effects of lowered voice pitch, to the memory-impairing effects of raised voice pitch, or to effects in both directions. What we observed was a pattern consistent with the presence of enhancing and impairing effects relative to the unmanipulated voice baseline condition (see Fig. 5). As was the case in Experiment 1, voice pitch effects were specific to men's voices, with no trace of any within-sex effects triggered by listening to women's voices. The preference data also replicated the pattern obtained in Experiment 1, and they showed in addition that the estimated strength of women's preferences for sexual dimorphism in men's voices depended on the particular pairing used on a preference trial. When manipulated male voices were compared-that is, on "standard" trials involving masculinised versus feminised voices, women's preferences for lowered pitch were significantly stronger 
than in "nonstandard" trials, where unmanipulated voices were judged against manipulated voices. This pattern most likely reflects the fact that the absolute pitch difference between recordings on the standard trials was equivalent to $40 \mathrm{~Hz}$, which was twice the $20-\mathrm{Hz}$ equivalent difference on the nonstandard trials.

\section{General discussion}

Nairne and Pandeirada (2008) argued that memory may be adapted to retain information related to fundamental adult behavioural "goals"- that is, to survive long enough to reproduce. We provide novel support for this particular functionalist framework, which so far has relied mainly on evidence of enhanced retention for information related to grassland survival (e.g., Kang et al., 2008; Klein et al., 2010; Nairne \& Pandeirada, 2008; Nairne, Thompson, \& Pandeirada, 2007; Weinstein et al., 2008; but see Soderstrom \& McCabe, 2011). We demonstrate here that women's memory is enhanced for information that co-occurs with male voices that exhibit a preferred lower pitch, as compared to less preferable raised-pitch male voices. These findings also complement work that appears to reveal potentially adaptive features of long-term memory for food location (New, Krasnow, Truxaw, \& Gaulin, 2007), spatial navigation more generally (e.g., Silverman et al., 2000), and future planning (Klein et al., 2010).

In both experiments, the effect of voice pitch on women's memory occurred for men's, but not for women's, voices, which suggests that the effect is not simply due to sex-typical vocal cues. The present studies therefore indicate for the first time, so far as we are aware, that signals from the opposite sex important for mate choice affect the accuracy of women's memory. It is worth noting that in a series of prior studies, Maner, Kenrick, and colleagues have repeatedly observed that while women do pay increased attention to attractive versus average male faces, these attentional enhancements do not appear to produce any detectable subsequent impact on memory for attractive versus average male faces (Anderson et al., 2010; Becker et al., 2005; Maner et al., 2003). To interpret this apparent disjunction between attention and memory, Anderson et al. recently suggested that physically attractive men may not be elaborately or intensively processed by women in the absence of further information about their social dominance and ability to accrue resources. Anderson et al. pointed out that such a pattern could reflect an efficient way to allocate cognitive effort within a mating strategy shaped by the potentially serious costs of becoming pregnant following a casual relationship (see Gangestad \& Simpson, 2000). If this reasoning is correct, one implication is that specific manipulations that target the "right" kind of cue may produce an effect on women's memory. Our findings here, albeit using voices not faces, reveal that sexual dimorphism is one such cue. Our findings are therefore consistent with, and to some degree supportive of, the proposals from Anderson et al.

Additional work will be required to identify specific cognitive mechanisms affected in women by the pitch of the male voice. We argue that in order to correctly discriminate studied versus unstudied objects, participants may have had to recollect particular details, colouring, perspective, and so forth. Hence, the effect of male voice pitch on women's memory might be linked to processes involved in the generation of episodic memories underlying the ability to recollect specific past experiences. Whether this is due to an effect on particular encoding mechanisms (e.g., of self-referential encoding), or to generalised arousal or attentional effects is unclear at the present time. Alternative explanations in terms of memory mechanisms may be viable, however-for example, in terms of the "familiarity" process invoked within dualprocess models of recognition (e.g., Yonelinas, 2002) or in terms of the fast unitisation of voice/object information (e.g., Bader, Mecklinger, Hoppstadter, \& Meyer, 2010). Our present findings do not allow us to distinguish between these various accounts, and this is a line of research that we are actively pursuing.

Returning finally to women's mate-choice behaviour, we argue that a memory adaptation sensitive to cues of sexual dimorphism could play an adaptive functional role within the comparative evaluation heuristic proposed by Bateson and Healy (2005). In particular, because of the doubleedged quality of male masculinity, it might be beneficial for women to treat information about the past behaviour of conspicuously masculine individuals as a decisive factor when judging whether or not they wish to have a relationship. Most notably, perhaps, the ability to recall details associated with particular masculine individuals may lead mate choice either towards or, indeed, away from that individual, depending on what exactly is remembered.

\section{Concluding remarks}

Tulving (2002) and Nairne and colleagues (e.g., Nairne \& Pandeirada, 2008; Nairne et al., 2007) have pointed out that we seldom ask how our memory systems developed into their modern form. In this article, we have focussed on a particular problem within women's mate choice (how to evaluate men in terms of their masculinity signals) that may have been solved by the development of a heuristic to compare currently available and absent alternative mates. Use of this heuristic entails a heavy demand upon memory, as the repository of information about the attributes and 
past behaviour of specific individuals. We were able to show that women's memory is in fact sensitive to masculinity cues in men's voice pitch. The present studies therefore make two main contributions. First of all, we extend prior work dealing with the perception of cues important for mate choice into a new cognitive domain, that of memory function. Secondly, our findings support key hypotheses of the functionalist framework (Nairne \& Pandeirada, 2008), which proposes that memory should be sensitive towards content of adaptive value and that this sensitivity should help us to act in ways that enhance our reproductive fitness.

Acknowledgments D. R. Feinberg is funded by the Social Sciences and Humanities Research Council of Canada. David S. Smith is supported by a donation from Mr. R. W. Smith.

\section{References}

Anderson, U. S., Perea, E. F., Becker, D. V., Ackerman, J. M., Shapiro, J. R., Neuberg, S. L., et al. (2010). I only have eyes for you: Ovulation redirects attention (but not memory) to attractive men. Journal of Experimental Social Psychology, 46, 804-8. doi:10.1016/j.jesp.2010.04.015

Apicella, C. L., \& Feinberg, D. R. (2009). Voice pitch alters matechoice-relevant perception in hunter-gatherers. Proceedings of the Royal Society B, 276, 1077-82.

Apicella, C. L., Feinberg, D. R., \& Marlowe, F. W. (2007). Voice pitch predicts reproductive success in male hunter-gatherers. Biology Letters, 3, 682-4.

Bader, R., Mecklinger, A., Hoppstadter, M., \& Meyer, P. (2010). Recognition memory for one-trial unitised word pairs: Evidence from event-related potentials. NeuroImage, 50, 772-81.

Bateson, M., \& Healy, S. D. (2005). Comparative evaluation and its implications for mate choice. Trends in Ecology \& Evolution, 20, 659-64.

Becker, V. D., Kenrick, D. T., Guerin, S., \& Maner, J. K. (2005). Concentrating on beauty: Sexual selection and socio-spatial memory. Personal and Social Psychology Bulletin, 31, 1643-52.

Boersma, P., \& Weenink, D. (2010). Praat: Doing phonetics by computer (Version 5103) [Computer program]. Available at www.praat.org

Booth, A., \& Dabbs, J. M. (1993). Testosterone and men's marriages. Social Forces, 72, 463-77.

Boothroyd, L. G., Jones, B. C., Burt, D. M., DeBruine, L. M. \& Perrett, D. I. (2008). Facial correlates of sociosexuality. Evolution and Human Behavior, 29(3), 211-218.

Childers, D. G., \& Wu, K. (1991). Gender recognition from speech, Part II: Fine analysis. Journal of the Acoustical Society of America, 90, 1841-56.

Collins, S. A. (2000). Men's voices and women's choices. Animal Behaviour, 60, 773-80. doi:10.1006/anbe.2000.1523

Dabbs, J. M., Jr., \& Mallinger, A. (1999). High testosterone levels predict low voice pitch among men. Personality and Individual Differences, 27, 801-4. doi:10.1016/S0191-8869(98)00272-4

Endler, J. A. (1986). Natural selection in the wild. Princeton, NJ: Princeton University Press.

Feinberg, D. R., Jones, B. C., Little, A. C., Burt, D. M., \& Perrett, D. I. (2005). Manipulations of fundamental and formant frequencies influence the attractiveness of human male voices. Animal Behaviour, 69, 561-8. doi:10.1016/j.anbehav.2004.06.012
Feinberg, D. R., Jones, B. C., Law Smith, M. J., Moore, F. R., DeBruine, L. M., Cornwell, R. E., et al. (2006). Menstrual cycle, trait estrogen level, and masculinity preferences in the human voice. Hormonal Behavior, 49, 215-22.

Feinberg, D. R., DeBruine, L. M., Jones, B. C., \& Little, A. C. (2008). Correlated preferences for men's facial and vocal masculinity. Evolution and Human Behavior, 29, 233-41. doi:10.1016/j. evolhumbehav.2007.12.008

Fink, B., \& Penton-Voak, I. (2002). Evolutionary psychology of facial attractiveness. Current Directions in Psychological Science, 11 (5), 154-8. doi:10.1111/1467-8721.00190

Gangestad, S. W., \& Simpson, J. A. (2000). The evolution of human mating: Trade-offs and strategic pluralism. The Behavioral and Brain Sciences, 23, 573-644. doi:10.1017/ S0140525X0000337X

Gangestad, S. W., \& Thornhill, R. (2003). Facial masculinity and fluctuating asymmetry. Evolution and Human Behavior, 24, 23141.

Hughes, S. M., Dispenza, F., \& Gallup, G. G., Jr. (2004). Ratings of voice attractiveness predict sexual behavior and body configuration. Evolution and Human Behavior, 25, 295-304. doi:10.1016/j. evolhumbehav.2004.06.001

Kang, S. H. K., McDermott, K. B., \& Cohn, S. M. (2008). The mnemonic advantage of processing fitness-relevant information. Memory \& Cognition, 36, 1151-6. doi:10.3758/MC.36.6.1151

Klein, S. B., Robertson, T. E., \& Delton, A. W. (2010). Facing the future: Memory as an evolved system for planning future acts. Memory \& Cognition, 38, 13-22. doi:10.3758/MC.38.1.13

Little, A. C., Jones, B. C., Waitt, C., Tiddeman, B. P., Feinberg, D. R., Perret, D. I., et al. (2008). Symmetry is related to sexual dimorphism in faces: Data across culture and species. PloS One, 3, 2106.

Maner, J. K., Kenrick, D. T., Becker, D. V., Delton, A. W., Hofer, B., Wilbur, C. J., et al. (2003). Sexually selective cognition: Beauty captures the mind of the beholder. Journal of Personality and Social Psychology, 85, 1107-20.

Nairne, J. S., \& Pandeirada, J. N. S. (2008). Adaptive memory: Remembering with a stone-age brain. Current Directions in Psychological Science, 17, 239-43. doi:10.1111/j.14678721.2008.00582.x

Nairne, J. S., Thompson, S. R., \& Pandeirada, J. N. S. (2007). Adaptive memory: Survival processing enhances retention. Journal of Experimental Psychology. Learning, Memory, and Cognition, 33, 263-73. doi:10.1037/0278-7393.33.2.263

New, J., Krasnow, M. M., Truxaw, D., \& Gaulin, S. J. C. (2007). Spatial adaptations for plant foraging: Women excel and calories count. Proceedings of the Royal Society B, 274, 2679-84.

Perrett, D. I., Lee, K. J., Penton-Voak, I., Rowland, D., Yoshikawa, S., Burt, D. M., et al. (1998). Effects of sexual dimorphism on facial attractiveness. Nature, 394, 884-7. doi:10.1038/29772

Puts, D. A. (2005). Mating context and menstrual phase affect women's preferences for male voice pitch. Evolution and Human Behavior, 26, 388-97. doi:10.1016/j.evolhumbehav.2005.03.001

Rhodes, G., Simmons, L. W., \& Peters, M. (2005). Attractiveness and sexual behavior: Does attractiveness enhance mating success? Evolution and Human Behavior, 26, 186-201.

Rhodes, G., Chan, J., Zebrowitz, L. A., \& Simmons, L. W. (2003). Does sexual dimorphism in human faces signal health? Proceedings of the Royal Society B, 270(Suppl), S93-5.

Schutzwohl, A., \& Koch, S. (2004). Sex differences in jealousy: The recall of cues to sexual and emotional infidelity in personally more and less threatening context conditions. Evolution and Human Behavior, 25, 249-57.

Sedikides, C., Ariely, D., \& Olsen, N. (1999). Contextual and procedural determinants of partner selection: Of asymmetric dominance and prominence. Social Cognition, 17, 118-39. 
Sherry, D. F., \& Schacter, D. L. (1987). The evolution of multiple memory systems. Psychological Review, 94, 439-54.

Silverman, I., Choi, J., Mackewn, A., Fisher, M., Moro, J., \& Olshansky, E. (2000). Evolved mechanisms underlying wayfinding: Further studies on the hunter-gatherer theory of spatial sex differences. Evolution and Human Behavior, 21, 201-13.

Soderstrom, N. C., \& McCabe, D. P. (2011). Are survival processing memory advantages based on ancestral priorities? Psychonomic Bulletin \& Review, 18, 564-9. doi:10.3758/s13423-011-0060-6

Traunmüller, H. (1990). Analytical expressions for the tonotopic sensory scale. Journal of the Acoustical Society of America, 88, 97-100. doi:10.1121/1.399849

Thornhill, R., \& Gangestad, S. W. (2006). Facial sexual dimorphism, developmental stability, and susceptibility to disease in men and women. Evolution and Human Behavior, 27, 131-44. doi:10.1016/j.evolhumbehav.2005.06.001

Tulving, E. (2002). Episodic memory: From mind to brain. Annual Review of Psychology, 53, 1-25.
Vukovic, J., Feinberg, D. R., Jones, B. C., DeBruine, L. M., Welling, L. L. M., Little, A. C., et al. (2008). Self-rated attractiveness predicts individual differences in women's preferences for masculine men's voices. Personality and Individual Differences, 45, 451-6. doi:10.1016/j.paid.2008.05.013

Vukovic, J., Jones, B. C., DeBruine, L., Feinberg, D. R., Smith, F. G., Little, A. C., et al. (2010). Women's own voice pitch predicts their preferences for masculinity in men's voices. Behavioral Ecology, 21, 767-72. doi:10.1093/beheco/arq051

Weinstein, Y., Bugg, J. M., \& Roediger, H. L., III. (2008). Can the survival recall advantage be explained by basic memory processes? Memory \& Cognition, 36, 913-919. doi:10.3758/MC.36.5.913

van Wingen, G., Mattern, C., Verkes, R. J., Buitelaar, J., \& Fernandez, G. (2008). Testosterone biases automatic memory processes in women towards potential mates. NeuroImage, 43, 114-20.

Yonelinas, A. P. (2002). The nature of recollection and familiarity: A review of 30 years of research. Journal of Memory and Language, 46, 441-517. 